\title{
Thrombembolic Events in Hospitalized COVID-19 Patients: What is the Role of the Sex?
}

\author{
Irit Nachtigall ${ }^{1,2}$ Sven Hohenstein ${ }^{2}$ Andreas Bollmann ${ }^{2}$ Marzia Bonsignore ${ }^{3}$ Daniela Husser ${ }^{2}$ \\ Ralf Kuhlen ${ }^{4}$ Andreas Meier Hellmann ${ }^{5}$
}

${ }^{1}$ Helios Kliniken Ost and Klinikum Emil-von-Behring, Berlin, Germany

${ }^{2}$ Heart Center Leipzig at University of Leipzig and Leipzig Heart Institute, Leipzig Germany

${ }^{3}$ Evangelische Kliniken Gelsenkirchen, Zentrum für

Krankenhaushygiene und Infektiologie, Gelsenkirchen, Germany

${ }^{4}$ Helios Health, Berlin, Germany

${ }^{5}$ Helios Kliniken GmbH, Berlin, Germany

TH Open 2021;5:e411-e414.

Over a year ago, the WHO declared COVID-19 a pandemic; from then on, all hopes were on the development of vaccines. So far, 4 vaccines have been approved in Europe. On March $11^{\text {th }}, 2021$, the European Medicines Agency (EMA) reported $\sim 30$ cases of thromboembolic events (TE) that were observed within 2 weeks after vaccinations with the AstraZeneca vaccine Vaxzevria, mostly being cerebral venous sinus thromboses in women younger than 60 years. Ca. 5 million people had received Vaxzevria in the EEA by then. Several European countries stopped their vaccinations with Vaxzevria temporarily.

COVID-19 infections increase the risk of developing TE. It has not yet been reported whether women develop more TE under Covid-19 than men. The aim of the present study was to determine the frequency, sex distribution and risk factors of TE among SARS-CoV-2 positive patients.

\section{Methods}

We analyzed claims data from 83 hospitals in the Helios Group. All patient 19,501 cases admitted between February $1^{\text {st }}, 2020$ and February $8^{\text {th }}, 2021$ with the International Statistical Classification of Diseases and Related Health Problems (ICD-10) code U07.1 (= PCR-confirmed infection with SARS-CoV-2), were included.

The following ICD-10 codes were used as definitions: Thrombocytopenia: D69.5, D69.6, pulmonary embolism: I26, thrombosis: I80, I81, I82, sinus vein thrombosis: G08, I67.6, I63.6. Only cases which were completed in the hospital, were included for hospital mortality $n 8,533$.

DOI https://doi.org/

10.1055/a-1585-9536.

ISSN 2512-9465.
Address for correspondence Irit Nachtigall, MD, Helios Kliniken Ost and Klinikum Emil-von-Behring, Walterhöfer Str 11, Berlin, Germany (e-mail: irit.nachtigall@helios-gesundheit.de).

The following ICD-10 codes were used as definitions: Thrombocytopenia: D69.5, D69.6, pulmonary embolism: I26, thrombosis: I80, I81, I82, sinus vein thrombosis: G08, I67.6, I63.6. Only cases which were completed in the hospital, were included for hospital mortality $(n=8,533)$.

We used the $\mathrm{R}$ software for statistical programming (version 4.0.2) for all analyses. The multivariable analyses of TE and in-hospital mortality were analyzed via logistic regression with log link function. In these models, we used sex, age (as numerical variable), comorbidities, and the frailty risk score ${ }^{1}$ as predictors; in the models for in-hospital mortality, TE was an additional predictor.

\section{Results}

19,501 patients aged $0-103 \mathrm{y}$ (median $74 \mathrm{y}, \mathrm{Q} 25=59 \mathrm{y}$, Q75 = 83y), 9,537 women (48.91\%) and 9,964 men (51.09\%) were included for the whole analysis, for calculation of the mortality 8,533 cases (85.64\%) were included. Patient characteristis of the total cohort and the subcohort with thromboembolic events are shown in - Table 1. At least one TE was coded in 963 patients (4.94\%) (433 pulmonary embolisms, 371 thrombocytopenias, 249 thromboses and 2 sinus vein thromboses, several events per patient being possible), incidence rate was 4,938 (per 100,000 cases; 95\% CI: 4640-5254). TE occurred in $4.94 \%$ of all inpatients; men were affected by $5.73 \%$ ( 571 / 9.964) and women by 4.11\% (392 / 9,537). The distribution of age and sex in thrmoboembolic events is shown in -Figure 1. In the multivariate regression analysis, independent risk factors for developing TE were among others male

(c) 2021. The Author(s).

This is an open access article published by Thieme under the terms of the Creative Commons Attribution License, permitting unrestricted use, distribution, and reproduction so long as the original work is properly cited. (https://creativecommons.org/licenses/by/4.0/)

Georg Thieme Verlag KG, Rüdigerstraße 14, 70469 Stuttgart, Germany 
e412 Thrombembolic Events in Hospitalized COVID-19 Nachtigall et al.

Table 1 Patient characteristics of the total cohort and the subcohort of patients with thromboembolic events (TE)

\begin{tabular}{|c|c|c|}
\hline \multirow[t]{2}{*}{ Group } & Total cohort & TE cohort \\
\hline & \multicolumn{2}{|l|}{ Proportion (n) } \\
\hline \multicolumn{3}{|l|}{ Sex } \\
\hline Male & $51.1 \%(9,964)$ & $59.3 \%(571)$ \\
\hline Female & $48.9 \%(9,537)$ & $40.7 \%(392)$ \\
\hline \multicolumn{3}{|l|}{ Age } \\
\hline Mean (SD) & $69.2 \pm 18.7$ & $69.9 \pm 14.4$ \\
\hline$\leq 17$ years & $1.5 \%(290)$ & $0.2 \%(2)$ \\
\hline $18-29$ years & $3.2 \%(616)$ & $0.8 \%(8)$ \\
\hline $30-39$ years & $3.9 \%(762)$ & $3.2 \%(31)$ \\
\hline $40-49$ years & $5.6 \%(1,099)$ & $4.5 \%(43)$ \\
\hline $50-59$ years & $11.5 \%(2,238)$ & $13.0 \%(125)$ \\
\hline $60-69$ years & $15.1 \%(2,952)$ & $21.8 \%(210)$ \\
\hline $70-79$ years & $21.7 \%(4,229)$ & $26.8 \%(258)$ \\
\hline $80-89$ years & $30.2 \%(5,894)$ & $24.8 \%(239)$ \\
\hline$\geq 90$ years & $7.3 \%(1,421)$ & $4.9 \%(47)$ \\
\hline \multicolumn{3}{|c|}{ Elixhauser comorbidity index } \\
\hline Mean (SD) & $10.6 \pm 11.2$ & $21.3 \pm 12.8$ \\
\hline$<0$ & $13.3 \%(2,586)$ & $2.5 \%(24)$ \\
\hline 0 & $17.3 \%(3,365)$ & $1.8 \%(17)$ \\
\hline $1-4$ & $5.4 \%(1,050)$ & $1.5 \%(14)$ \\
\hline$\geq 5$ & $64.1 \%(12,500)$ & $94.3 \%(908)$ \\
\hline \multicolumn{3}{|c|}{ Congestive heart failure } \\
\hline no & $76.2 \%(14,863)$ & $68.0 \%(655)$ \\
\hline yes & $23.8 \%(4,638)$ & $32.0 \%(308)$ \\
\hline \multicolumn{3}{|c|}{ Cardiac arrhythmias } \\
\hline no & $74.1 \%(14,443)$ & $68.8 \%(663)$ \\
\hline yes & $25.9 \%(5,058)$ & $31.2 \%(300)$ \\
\hline \multicolumn{3}{|l|}{ Valvular disease } \\
\hline no & $92.6 \%(18,052)$ & $89.5 \%(862)$ \\
\hline yes & $7.4 \%(1,449)$ & $10.5 \%(101)$ \\
\hline \multicolumn{3}{|c|}{ Pulmonary circulation disorders } \\
\hline no & $95.3 \%(18,594)$ & $52.6 \%(507)$ \\
\hline yes & $4.7 \%(907)$ & $47.4 \%(456)$ \\
\hline \multicolumn{3}{|c|}{ Peripheral vascular disorders } \\
\hline no & $92.6 \%(18,054)$ & $90.4 \%(871)$ \\
\hline yes & $7.4 \%(1,447)$ & $9.6 \%(92)$ \\
\hline \multicolumn{3}{|c|}{ Hypertension, uncomplicated } \\
\hline no & $56.2 \%(10,955)$ & $59.4 \%(572)$ \\
\hline yes & $43.8 \%(8,546)$ & $40.6 \%(391)$ \\
\hline \multicolumn{3}{|c|}{ Hypertension, complicated } \\
\hline no & $88.5 \%(17,265)$ & $85.9 \%(827)$ \\
\hline yes & $11.5 \%(2,236)$ & $14.1 \%(136)$ \\
\hline
\end{tabular}

Table 1 (Continued)

\begin{tabular}{|c|c|c|}
\hline \multirow[t]{2}{*}{ Group } & Total cohort & TE cohort \\
\hline & \multicolumn{2}{|l|}{ Proportion (n) } \\
\hline \multicolumn{3}{|c|}{ Paralysis } \\
\hline no & $95.1 \%(18,552)$ & $95.2 \%(917)$ \\
\hline yes & $4.9 \%(949)$ & $4.8 \%(46)$ \\
\hline \multicolumn{3}{|c|}{ Other neurological disorders } \\
\hline no & $91.2 \%(17,785)$ & $89.9 \%(866)$ \\
\hline yes & $8.8 \%(1,716)$ & $10.1 \%(97)$ \\
\hline \multicolumn{3}{|c|}{ Chronic pulmonary disease } \\
\hline no & $88.5 \%(17,267)$ & $89.8 \%(865)$ \\
\hline yes & $11.5 \%(2,234)$ & $10.2 \%(98)$ \\
\hline \multicolumn{3}{|c|}{ Diabetes, uncomplicated } \\
\hline no & $83.2 \%(16,221)$ & $80.9 \%(779)$ \\
\hline yes & $16.8 \%(3,280)$ & $19.1 \%(184)$ \\
\hline \multicolumn{3}{|c|}{ Diabetes, complicated } \\
\hline no & $88.4 \%(17,231)$ & $88.7 \%(854)$ \\
\hline yes & $11.6 \%(2,270)$ & $11.3 \%(109)$ \\
\hline \multicolumn{3}{|c|}{ Hypothyroidism } \\
\hline no & $87.7 \%(17,109)$ & $88.6 \%(853)$ \\
\hline yes & $12.3 \%(2,392)$ & $11.4 \%(110)$ \\
\hline \multicolumn{3}{|c|}{ Renal failure } \\
\hline no & $69.0 \%(13,460)$ & $70.6 \%(680)$ \\
\hline yes & $31.0 \%(6,041)$ & $29.4 \%(283)$ \\
\hline \multicolumn{3}{|c|}{ Liver disease } \\
\hline no & $96.0 \%(18,725)$ & $87.7 \%(845)$ \\
\hline yes & $4.0 \%(776)$ & $12.3 \%(118)$ \\
\hline \multicolumn{3}{|c|}{ Peptic ulcer disease excluding bleeding } \\
\hline no & $99.9 \%(19,487)$ & $99.8 \%(961)$ \\
\hline yes & $0.1 \%(14)$ & $0.2 \%(2)$ \\
\hline \multicolumn{3}{|c|}{ AIDS/HIV } \\
\hline no & $100.0 \%(19,494)$ & $99.9 \%(962)$ \\
\hline yes & $0.0 \%(7)$ & $0.1 \%(1)$ \\
\hline \multicolumn{3}{|c|}{ Lymphoma } \\
\hline no & $99.3 \%(19,358)$ & $97.0 \%(934)$ \\
\hline yes & $0.7 \%(143)$ & $3.0 \%(29)$ \\
\hline \multicolumn{3}{|c|}{ Metastatic cancer } \\
\hline no & $97.7 \%(19,044)$ & $95.2 \%(917)$ \\
\hline yes & $2.3 \%(457)$ & $4.8 \%(46)$ \\
\hline \multicolumn{3}{|c|}{ Solid tumor without metastasis } \\
\hline no & $95.1 \%(18,547)$ & $92.3 \%(889)$ \\
\hline yes & $4.9 \%(954)$ & $7.7 \%(74)$ \\
\hline \multicolumn{3}{|c|}{ Rheumatoid artritis/collaged vascular disease } \\
\hline no & $98.2 \%(19,157)$ & $97.6 \%(940)$ \\
\hline yes & $1.8 \%(344)$ & $2.4 \%(23)$ \\
\hline
\end{tabular}


Table 1 (Continued)

\begin{tabular}{|c|c|c|}
\hline \multirow[t]{2}{*}{ Group } & Total cohort & TE cohort \\
\hline & \multicolumn{2}{|l|}{ Proportion (n) } \\
\hline \multicolumn{3}{|c|}{ Coagulopathy } \\
\hline no & $95.5 \%(18,625)$ & $57.7 \%(556)$ \\
\hline yes & $4.5 \%(876)$ & $42.3 \%(407)$ \\
\hline \multicolumn{3}{|c|}{ Obesity } \\
\hline no & $88.3 \%(17,216)$ & $85.6 \%(824)$ \\
\hline yes & $11.7 \%(2,285)$ & $14.4 \%(139)$ \\
\hline \multicolumn{3}{|c|}{ Weight loss } \\
\hline no & $89.4 \%(17,436)$ & $80.0 \%(770)$ \\
\hline yes & $10.6 \%(2,065)$ & $20.0 \%(193)$ \\
\hline \multicolumn{3}{|c|}{ Fluid and electrolyte disorders } \\
\hline no & $57.4 \%(11,198)$ & $42.8 \%(412)$ \\
\hline yes & $42.6 \%(8,303)$ & $57.2 \%(551)$ \\
\hline \multicolumn{3}{|c|}{ Blood loss anemia } \\
\hline no & $99.5 \%(19,403)$ & $98.5 \%(949)$ \\
\hline yes & $0.5 \%(98)$ & $1.5 \%(14)$ \\
\hline \multicolumn{3}{|c|}{ Deficiency anemia } \\
\hline no & $96.6 \%(18,845)$ & $94.7 \%(912)$ \\
\hline yes & $3.4 \%(656)$ & $5.3 \%(51)$ \\
\hline \multicolumn{3}{|c|}{ Alcohol abuse } \\
\hline no & $98.1 \%(19,134)$ & $96.2 \%(926)$ \\
\hline yes & $1.9 \%(367)$ & $3.8 \%(37)$ \\
\hline \multicolumn{3}{|c|}{ Drug abuse } \\
\hline no & $99.6 \%(19,430)$ & $99.9 \%(962)$ \\
\hline yes & $0.4 \%(71)$ & $0.1 \%(1)$ \\
\hline \multicolumn{3}{|c|}{ Psychoses } \\
\hline no & $98.7 \%(19,256)$ & $99.1 \%(954)$ \\
\hline yes & $1.3 \%(245)$ & $0.9 \%(9)$ \\
\hline \multicolumn{3}{|c|}{ Depression } \\
\hline no & $94.0 \%(18,334)$ & $94.6 \%(911)$ \\
\hline yes & $6.0 \%(1,167)$ & $5.4 \%(52)$ \\
\hline
\end{tabular}

sex, lymphomas, liver diseases and congestive heart failure (-Table 2). TE were associated with an increased risk of death; the mortality rate was $20.7 \%$ in the group without TE and $39.8 \%$ in the group with such an event. (OR 2.28; 95\% $\mathrm{Cl} 1.93-2.70$ ).

\section{Discussion}

In our cohort of Covid-19 inpatients, TE occurred in approx. $5 \%$; involved mostly pulmonary embolisms and affected mainly men in their 60ies. In addition to various pre-existing conditions, we found the male sex to be a major independent risk factor for the development of TE. TE are a common complication of COVID-19 and have been reported to occur in ca. $7 \%$ of inpatients treated with thromboembolism prophy-
Table 2 Results of multivariable analyses of thromboembolic complications

$\left.\begin{array}{|l|l|l|}\hline \text { Variable } & \text { OR }(95 \% \mathrm{CI}) & P \text { value } \\ \hline \text { Age } & 1.00(0.99-1.00) & 0.10 \\ \hline \text { Female sex } & 0.73(0.63-0.83) & <0.01 \\ \hline \text { Frailty risk score } & 1.04(1.02-1.05) & <0.01 \\ \hline \text { Congestive heart failure } & 1.40(1.17-1.68) & <0.01 \\ \hline \text { Cardiac arrhythmias } & 1.06(0.90-1.24) & 0.51 \\ \hline \text { Valvular disease } & 1.22(0.96-1.54) & 0.10 \\ \hline \begin{array}{l}\text { Peripheral vascular } \\ \text { disorders }\end{array} & 1.07(0.84-1.36) & 0.59 \\ \hline \begin{array}{l}\text { Hypertension, } \\ \text { uncomplicated }\end{array} & 0.81(0.69-0.94) & <0.01 \\ \hline \begin{array}{l}\text { Hypertension, } \\ \text { complicated }\end{array} & 0.87(0.69-1.11) & 0.26 \\ \hline \text { Paralysis } & 0.67(0.48-0.93) & 0.02 \\ \hline \begin{array}{l}\text { Other neurological } \\ \text { disorders }\end{array} & 0.92(0.73-1.16) & 0.47 \\ \hline \begin{array}{l}\text { Chronic pulmonary } \\ \text { disease }\end{array} & 0.75(0.60-0.93) & <0.01 \\ \hline \text { Diabetes, uncomplicated } & 1.05(0.89-1.26) & 0.55 \\ \hline \text { Diabetes, complicated } & 0.85(0.67-1.07) & 0.18 \\ \hline \text { Hypothyroidism } & 0.93(0.75-1.15) & 0.48 \\ \hline \text { Renal failure } & 0.66(0.56-0.78) & <0.01 \\ \hline \text { Liver disease } & 2.94(2.33-3.70) & <0.01 \\ \hline \text { AlDS/HIV } & 2.36(0.27-20.89) & 0.44 \\ \hline \text { Lymphoma } & 5.19(3.36-8.00) & <0.01 \\ \hline \text { Metastatic cancer } & 1.65(1.07-2.55) & 0.02 \\ \hline \begin{array}{l}\text { Solid tumor without } \\ \text { metastasis }\end{array} & 1.22(0.86-1.71) & 0.26 \\ \hline \text { Rheumatoid artritis/ } \\ \text { collaged vascular disease }\end{array}\right)$

Quality of regression model tested with the Hosmer-Lemeshow test, indicating good calibration $\left(X^{2}=23.556 ; p=0.428\right)$.

laxis. $^{2}$ Several possible pathomechanisms have been discussed, including a direct endothelial damage ${ }^{3}$ as well as an antibody-mediated activation of platelets via the Fcr-IIa receptor. ${ }^{4}$ Men are at an increased risk of a severe course of Covid- $19^{5}$; TE are presumably part of this multifactorial, gender-specific risk.

The antibody-mediated activation of platelets via the FcrIla receptor has also been suggested as pathomechanism for TE after Vaxzevria. ${ }^{6}$ It is unclear, why younger women seem 


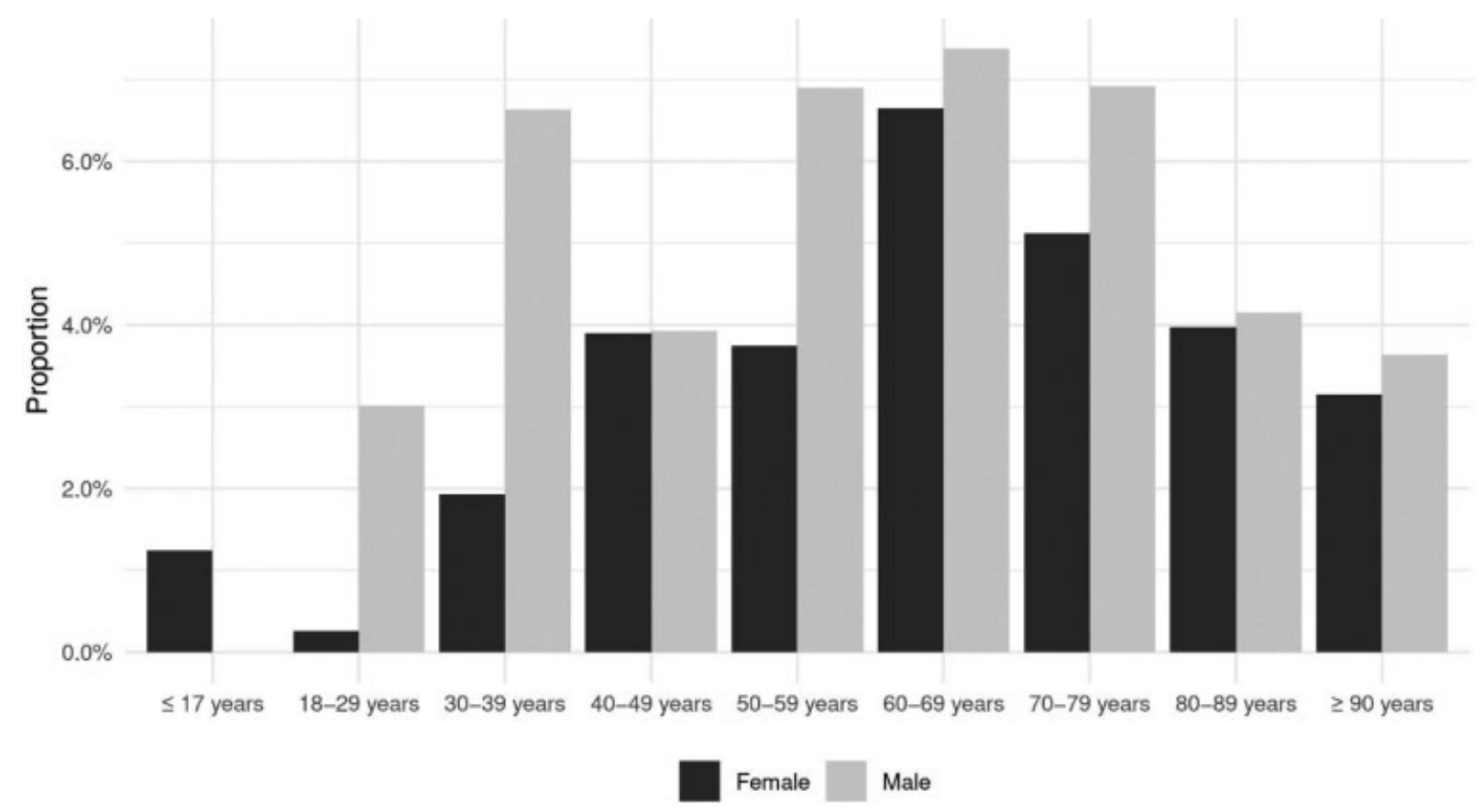

Fig. 1 Proportion of patients with at least one thromboembolic event in Covid-19 inpatients by age and sex.

to be affected more often by this complication. In general, sinus vein thromboses occur mostly in younger women. ${ }^{7}$ The EMA reviewed 62 cases of cerebral venous sinus and 24 of splanchnic vein thromboses reported until March 22th, 2021. Although most of the cases reported have occurred in women $<60 \mathrm{y}$, no specific risk factors like sex or age were confirmed; the risk for TE after the vaccinations was estimated at 1: 100000 .

In summary, we found that a large number of Covid-19 inpatients have thromboembolic complications, at a frequency that is 5000 times higher than the one of TE after Vaxzevria. Although similar pathomechanisms have been discussed for both, the development of TE in Covid-19 and after vaccination, these two phenomena differ clearly in the type of thromboses that occur and in the sex distribution. The observational design of our study based on claims data are a strong limitation; at best, the results can provide a signal, especially information on medications and their different impact on both sexes need to be addressed. To our opinion, the sex aspect of thromboembolic events in Covid-19 and after Vaxzevria has not been adequately addressed so far and needs further investigation.

\section{Conflict of Interest}

RK declares to hold shares of Fresenius, all other authors declare not to have any conflicts of interest.

\section{References}

1 Gilbert T, Neuburger J, Kraindler J, et al. Development and validation of a Hospital Frailty Risk Score focusing on older people in acute care settings using electronic hospital records: an observational study. Lancet 2018;391(10132):1775-1782. Doi: 10.1016/S0140-6736(18)30668-8

2 Aktaa S, Wu J, Nadarajah R, et al. Incidence and mortality due to thromboembolic events during the COVID-19 pandemic: Multisourced population-based health records cohort study. Thromb Res 2021;202:17-23. Doi: 10.1016/j.thromres.2021.03.006

3 Harenberg J, Violi F. Waves of SARS-CoV-2 Infection and Blood Coagulation-A Link and Beyond. Thromb Haemost 2021;121(01): 4-6

4 Althaus K, Marini I, Zlamal J, et al. Antibody-induced procoagulant platelets in severe COVID-19 infection. Blood 2021;137(08): 1061-1071. Doi: 10.1182/blood.2020008762

5 Nachtigall I, Lenga P, Jóźwiak K, et al. Clinical course and factors associated with outcomes among 1904 patients hospitalized with COVID-19 in Germany: an observational study. Clin Microbiol Infect 2020;26(12):1663-1669. Doi: 10.1016/j.cmi.2020.08.011

6 Greinacher A, Thiele T, Warkentin TE, Weisser K, Kyrle PA, Eichinger S. Thrombotic Thrombocytopenia after ChAdOx1 nCov-19 Vaccination. N Engl J Med 2021;384(22):2092-2101. Doi: 10.1056/NEJMoa2104840

7 Ferro JM, Canhão P, Stam J, Bousser MG, Barinagarrementeria FISCVT Investigators. Prognosis of cerebral vein and dural sinus thrombosis: results of the International Study on Cerebral Vein and Dural Sinus Thrombosis (ISCVT). Stroke 2004;35(03): 664-670 